\title{
Sharing - Vom Horten und Hüten zum Teilen und Tauschen
}

\author{
Josephine Hofmann
}

Eingegangen: 17. Oktober 2014 / Angenommen: 22. Oktober 2014

(C) Springer Fachmedien Wiesbaden 2014

Sharing - ein sehr alter Begriff in der Wirtschaftsinformatik: Es geht darum, eine Ressource mit Hilfe von Informations- und Kommunikationstechnologien gemeinsam zu nutzen. Schon in den Anfängen der Informatik wurden unterschiedliche Sharing-Konzepte z. B. zur Nutzung von Rechnerzeit oder Speicherplatz eingeführt. Später wurden Softwarelösungen unter dem Begriff Open Source entwickelt und frei verfügbar gemacht. Im heutigen Zeitalter des elektronischen Geschäfts, d. h. bei digitalen Produkten und Dienstleistungen, wird das Teilen beinahe unbeschränkt möglich. Formen wie Social Commerce lassen alle Beteiligten am Erfolg des Geschäfts partizipieren. Aber auch in der Freizeit sind Sharing-Plattformen für Fotos, Musik oder Filme kaum mehr wegzudenken. Unabhängig vom engeren Fokus der IT geben Zukunftsforscher dem Sharing-Prinzip sogar die Bedeutung, letztlich das treibende und neue Paradigma unseres Wirtschaftssystems zu werden.

Der folgende Beitrag zeichnet ein hochinteressantes Fachgespräch ausgewiesener und sehr erfahrener Wissenschaftler im Bereich der Wirtschaftsinformatik nach. David Gugerli, Andreas Meier, Carl August Zehnder und Daniela Zetti tauschen sich, mit sehr interessanten Rückblicken und Einschätzungen, über zentrale Konzeptumsetzungen des „Sharing-Prinzips“ in der Geschichte der Informatik aus: Die Zeitreise startet bei Time-Sharing-Konzepten und schreitet über Storage Sharing über das Sharen von personellen Ressourcen bis hin zum Sharing von sozialen Kontakten fort. Ein sehr lesenswerter Beitrag, der auch belegt, dass viele Konzeptbestandteile schon früh geboren wurden und mit der Verfügbarmachung von leistungsfähigen Rechnerund Netzkomponenten Schritt für Schritt den Bedeutungsumfang von Rechnern für

\footnotetext{
J. Hofmann $(\bowtie)$

Fraunhofer IAO,

Nobelstraße 12,

70569 Stuttgart, Deutschland

E-Mail: josephine.hofmann@iao.fraunhofer.de
} 
unser Leben stark verbreitert haben. Der Beitrag kommt im Finale aber auch zu einigen sehr nachdenklichen Schlüssen, die wir für sehr lesenswert halten.

Josephine Hofmann 